\title{
Investigation of the Convergence Behavior with Fluctuation Features in the Fourier Modal Analysis of a Metallic Grating
}

\author{
Hwi Kim ${ }^{1}$, Gwanwoo Park ${ }^{1}$, and Changsoon Kim ${ }^{2,3 *}$ \\ ${ }^{1}$ Department of Electronics and Information Engineering, College of Science and Technology, \\ Korea University, Sejong Campus, Sejong 339-700, Korea \\ ${ }^{2}$ Graduate School of Convergence Science and Technology, Seoul National University, \\ 1 Gwanak-ro, Gwanak-gu, Seoul 151-742, Korea \\ ${ }^{3}$ Advanced Institutes of Convergence Technology, Iui-dong, Yeongtong-gu, Suwon 443-270, Korea
}

(Received August 27, 2012 : revised September 4, 2012 : accepted September 5, 2012)

\begin{abstract}
We observe that the transmission and reflection efficiencies of a one-dimensional metallic grating under transverse-magnetic illumination calculated using the Fourier modal method (FMM) with the Fourier factorization rules have peculiar fluctuations, albeit small in magnitude, as the number of field harmonics increases. It is shown that when the number of Fourier terms for the electromagnetic field is increased from that in the conventional FMM, the fluctuations due to non-convergent highly evanescent eigenmodes can be eliminated. Our examination reveals that the fluctuations originate from the Gibbs phenomenon inherent in the Fourier-series representation of a permittivity function with discontinuities, and from non-convergence of highly evanescent internal Bloch eigenmodes.
\end{abstract}

Keywords : Fourier modal method, Rigorous coupled wave analysis, Surface plasmons, Gratings OCIS codes : (050.2770) Gratings; (050.6624) Subwavelength structures; (240.6680) Surface plasmons

\section{INTRODUCTION}

The Fourier modal method (FMM) is one of the most popular numerical electromagnetic analysis methods for periodic optical structures [1]. With recent developments of the initial theoretical framework, such as perfect matched layer (PML) $[2,3]$ and the generalized scattering-matrix method $[4,5]$, the range of applications of the FMM was extended to include aperiodic, anisotropic device structures and more generalized photonic network structures [5]. Furthermore, with the performance improvement of modern computers, the FMM has become a crucial tool in the development of various optical and optoelectronic devices, such as anti-reflection coatings [6], broadband reflectors [7, 8], narrowband bandpass filters [8], solar cells [9], waveguides [2, 10], and optomechanical devices [11].

Perhaps the most important development in the history of the FMM is Li's Fourier factorization rule for improving the convergence of the FMM in analyzing metallic gratings under transverse-magnetic (TM) polarization [12-14]. Since the FMM with Li's factorization rule shows good convergence in analyzing such structures, it has become one of the most important analysis tools in the field of plasmonics $[15,16]$. However, through FMM analyses of metallic structures under surface plasmon resonance (SPR), we have found that the coefficients of transmission and reflection have peculiar small fluctuations when plotted versus the number of Fourier terms used, even though the FMM has a large number of Fourier terms and is featured with Li's factorization rule.

This motivates the investigation of the origins of the fluctuations observed in the FMM analysis of a one-dimensional lamellar metallic grating with discontinuities in the permittivity profile. An in-depth discussion on the internal modal structure of the FMM and the Gibbs phenomenon arising in the Fourier representation of discontinuous metallic structures is presented. To systematically study this problem, we modify the conventional formulation of the FMM to control the number of Fourier harmonics representing the fields independently of that of Fourier terms associated with the permittivity profile, and examine the respective effects of the numbers of the field and structure harmonics

\footnotetext{
*Corresponding author: changsoon@snu.ac.kr

Color versions of one or more of the figures in this paper are available online.
} 
on the convergence behavior of the FMM. Based on the analysis, we discuss the origins of the fluctuations in the convergence of the FMM, and conclude with perspective and a future plan for enhancing the accuracy of the FMM.

This paper is organized as follows. In Sec. 2, the numerical framework of the FMM is reviewed. In Sec. 3, the main problem of interest is stated with an example of the FMM analysis. The reason for the problem is explained within the conventional FMM framework by discussing the Gibbs phenomenon and its physical relation to fluctuations. In Sec. 4, we modify the conventional FMM by de-coupling the linkage between the numbers of the field and structure harmonics, examine the resulting convergence behaviors of reflection and transmission efficiencies of a metallic grating, and clarify the origins of the fluctuations, followed by concluding remarks in Sec. 5 .

\section{NUMERICAL FRAMEWORK OF THE FOURIER MODAL METHOD}

Before investigating the convergence behaviors of the FMM for electromagnetic simulations of a metallic grating structure, we briefly review the numerical framework of the FMM [1]. For a one-dimensional grating structure investigated in the following sections (Fig. 1), the permittivity in the grating layer, $\varepsilon(x)$, is approximated by a truncated Fourier series, $\hat{\varepsilon}(x)$ :

$$
\hat{\varepsilon}(x)=\sum_{m=-2 M}^{2 M} \varepsilon_{m} e^{j m G x},
$$

where $G=2 / \Lambda$, and $\Lambda$ is the grating period. In the FMM, the internal electric and magnetic eigenmodes, $\mathbf{E}^{(g)}$ and $\mathbf{H}^{(g)}$, in the grating structure take the form of a truncated pseudo-Fourier series:

$$
\begin{aligned}
{\left[\begin{array}{l}
\mathbf{E}^{(g)} \\
\mathbf{H}^{(g)}
\end{array}\right] } & =\left[\begin{array}{lll}
E_{x}^{(g)} & E_{y}^{(g)} & E_{z}^{(g)} \\
H_{x}^{(g)} & H_{y}^{(g)} & H_{z}^{(g)}
\end{array}\right] \\
& =e^{j k^{(g)} z} e^{j\left(k_{x} x+k_{y} y\right)} \sum_{m=-M}^{M}\left[\begin{array}{lll}
\left(E_{x}^{(g)}\right)_{m} & \left(E_{y}^{(g)}\right)_{m} & \left(E_{z}^{(g)}\right)_{m} \\
\left(H_{x}^{(g)}\right)_{m} & \left(H_{y}^{(g)}\right)_{m} & \left(H_{z}^{(g)}\right)_{m}
\end{array}\right] e^{j m G x},
\end{aligned}
$$

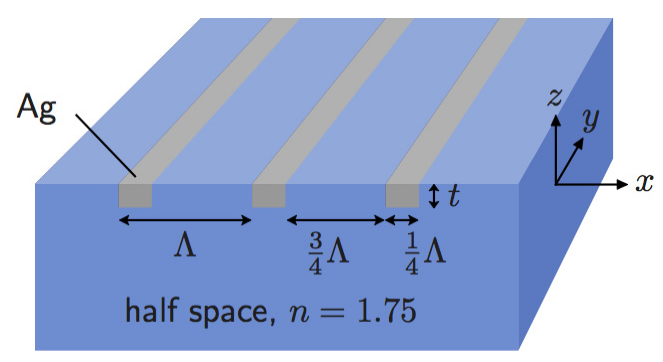

FIG. 1. Schematic of a one-dimensional metallic grating embedded in a substrate occupying the lower half space. where $e^{j k^{(g)} z}$ is the Bloch eigen-phase term, $\left(E_{x}^{(g)}\right)_{m},\left(E_{y}^{(g)}\right)_{m}$, $\left(E_{z}^{(g)}\right)_{m}$ and $\left(H_{x}^{(g)}\right)_{m}, \quad\left(H_{y}^{(\mathrm{g})}\right)_{m}, \quad\left(H_{z}^{(\mathrm{g})}\right)_{m}$ are the Fourier coefficients of the electric and magnetic field envelope functions, respectively, and $k_{x}$ and $k_{y}$ are determined by the wave vector of the incident plane wave. The Fourier coefficients and Bloch eigen-phase terms are calculated by substituting Eqs. (1) and (2) into Maxwell's equations and solving the resulting algebraic eigenvalue equation [1]. In doing so for TM-polarized (magnetic field parallel to the $y$-axis) waves, the Fourier coefficients of the axial (z-directional) electric displacement vector, $\varepsilon(x) E_{z}^{(g)}$, are given by the Laurent's rule:

$$
\left(\varepsilon E_{z}^{(g)}\right)_{l}=\sum_{m=-M}^{M} \llbracket \varepsilon \rrbracket_{l, m}\left(E_{z}^{(g)}\right)_{m}
$$

where $\llbracket \varepsilon \rrbracket_{l, m}$ are the $(l, m)$ matrix element of the Toeplitz matrix $\llbracket \varepsilon \rrbracket$ generated from the Fourier coefficients of $\varepsilon(x)$, $\varepsilon_{m}$. The Fourier coefficients of the transversal (x-directional) electric displacement vector, $\varepsilon(x) E_{x}^{(g)}$, are obtained in a different fashion, known as Li's rule [12, 13]:

$$
\left(\varepsilon E_{x}^{(g)}\right)_{l}=\sum_{m=-M}^{M} \llbracket 1 / \varepsilon \rrbracket_{l, m}^{-1}\left(E_{x}^{(g)}\right)_{m}
$$

where $\llbracket 1 / \varepsilon \rrbracket_{l, m}^{-1}$ are the $(l, m)$ matrix elements of the inverse of the Toeplitz matrix generated from the Fourier coefficients of $1 / \varepsilon(x)$. Li's factorization rules are critical for fast convergence of the FMM for the case of metallic gratings under TM illumination [13]. In the case of transverse-electric (TE, electric field parallel to the $y$-axis) waves, the Fourier coefficients of $\varepsilon(x) E_{y}^{(g)}$ are obtained as in Eq. (3).

In the conventional formulation of the FMM, the $4(2 M+1)$ internal Bloch eigenmodes are obtained numerically, and the total electromagnetic field inside the grating structure is described by the coherent superposition of $4(2 M+1)$ internal Bloch eigenmodes as

$$
\left[\begin{array}{l}
\mathbf{E} \\
\mathbf{H}
\end{array}\right]=\sum_{g=1}^{4(2 M+1)} C_{g}\left[\begin{array}{l}
\mathbf{E}^{(g)} \\
\mathbf{H}^{(g)}
\end{array}\right],
$$

where $C_{g}$ is the coupling coefficient that represents the degree of coupling between the external excitation and the $g^{\text {th }}$ internal electromagnetic Bloch eigenmode, and is matched by electromagnetic boundary conditions.

\section{FOURIER MODAL ANALYSIS OF A ONE-DIMENSIONAL METALLIC GRATING}

A grating structure that we analyze to examine the convergence behaviors of the FMM is illustrated in Fig. 1. An optical plane wave from the free-space region is 


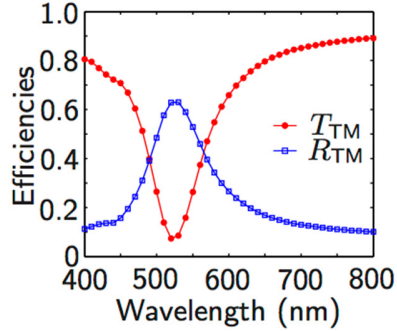

(a)

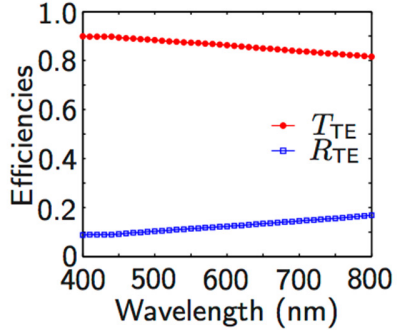

(b)
FIG. 2. (a) Transmission $\left(T_{\mathrm{TM}}\right)$ and reflection $\left(R_{\mathrm{TM}}\right)$ efficiencies for TM-polarized illumination. (b) Transmission $\left(T_{\mathrm{TE}}\right)$ and reflection $\left(R_{\mathrm{TE}}\right)$ efficiencies for TE-polarized illumination.

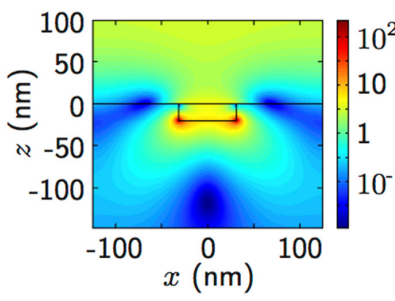

(a)

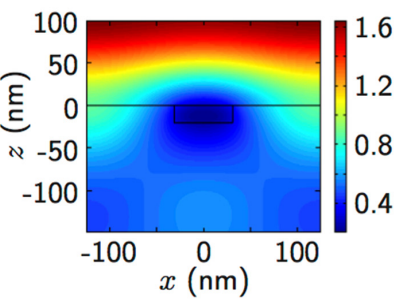

(b)
FIG. 3. The electric field intensity profiles for TM (a) and TE (b) polarization when $\lambda=520 \mathrm{~nm}$.

incident normal to the $x y$ plane on a substrate with an index of refraction $(n)$ of 1.75 , occupying the lower half space, in which a $\mathrm{Ag}$ periodic binary grating, with a period ( $\Lambda$ ) of $250 \mathrm{~nm}$, a fill-factor of 0.25 , and a thickness of $20 \mathrm{~nm}$, is embedded. Figure 2(a) shows the spectra of external transmission and reflection efficiencies $\left(T_{\mathrm{TM}}\right.$ and $R_{\mathrm{TM}}$ ), calculated using the FMM, for a TM-polarized incident plane wave, whose wavelength $\lambda$ is varied from $400 \mathrm{~nm}$ to $800 \mathrm{~nm}$. The results calculated for a TE-polarized plane wave ( $T_{\mathrm{TE}}$ and $\left.R_{\mathrm{TE}}\right)$ are shown in Fig. 2(b). The calculations were performed with $M=500$, and the complex permittivity of $\mathrm{Ag}$ is taken from the literature [17].

The $R_{\mathrm{TM}}$ spectrum has the main peak at $\lambda=520 \mathrm{~nm}$ and a small shoulder at $\lambda=420 \mathrm{~nm}$, both of which are attributed to SPRs. In contrast, the $T_{\mathrm{TE}}$ and $R_{\mathrm{TE}}$ spectra are nearly featureless [Fig. 2(b)]. In Figs. 3(a) and 3(b), the $\mathrm{TM}$ and TE electric field intensity profiles for $\lambda=520 \mathrm{~nm}$ are shown, respectively. For TM polarization, the electric field is tightly concentrated on the sidewalls and the bottom surface of the grating due to SPRs. In contrast, the field enhancements near the grating boundaries are not observed for the case of TE polarization, as shown in Fig. 3(b).

Next, we investigate the convergence characteristics of the FMM calculation. In Fig. 4, $T_{\mathrm{TM}}[(\mathrm{a})], R_{\mathrm{TM}}[(\mathrm{b})], T_{\mathrm{TE}}$ [(c)], and $R_{\mathrm{TE}}[(\mathrm{d})]$ are plotted as a function of $M$ for the three selected wavelengths, $420 \mathrm{~nm}$ (blue), $520 \mathrm{~nm}$ (red), and $750 \mathrm{~nm}$ (black). For the TM case, the results for all three wavelengths seem to be converged for sufficiently large $M(>100)$ but close examinations reveal that they
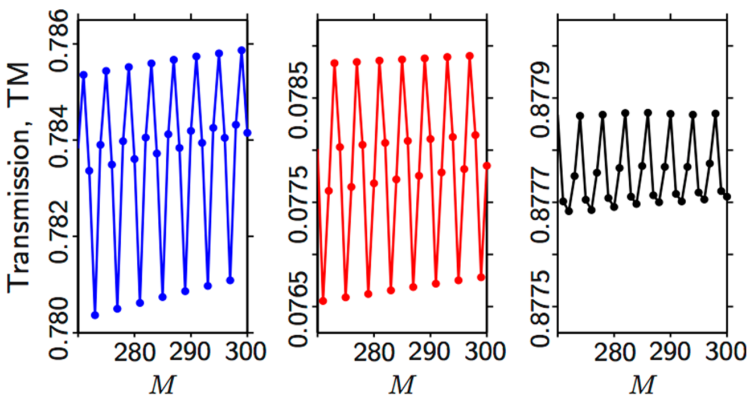

(a)
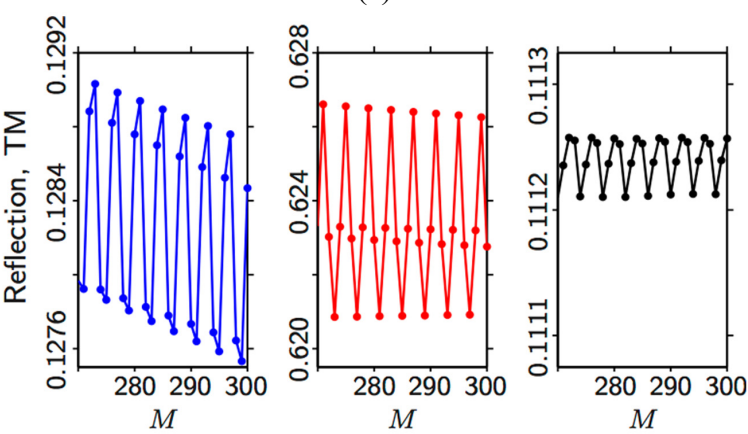

(b)
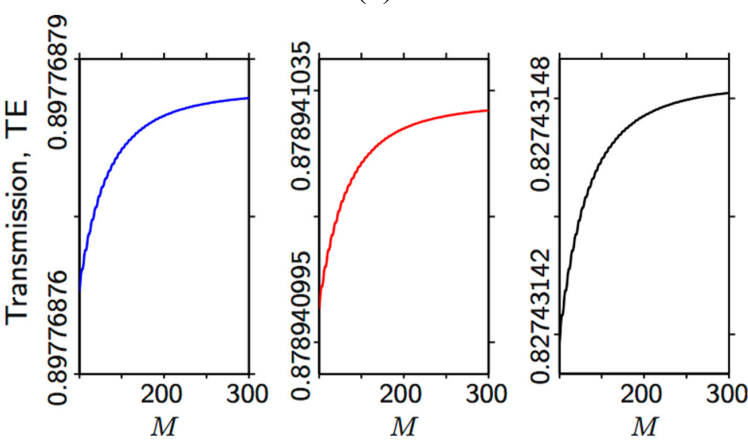

(c)
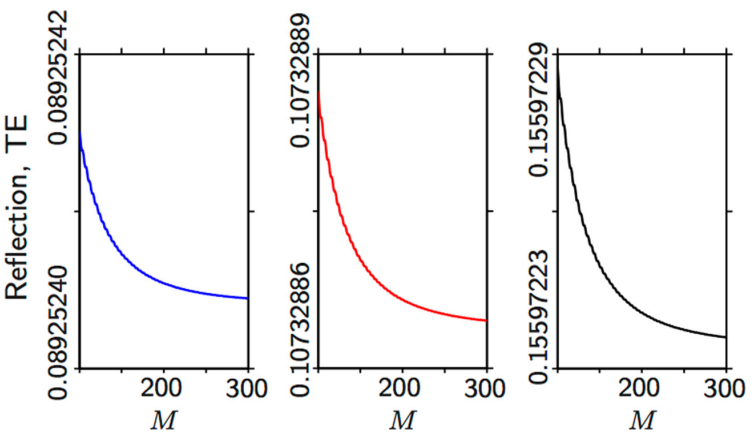

(d)

FIG. 4. Convergence behavior of transmission and reflection efficiencies for TM- and TE-polarized plane waves with $\lambda=$ $420 \mathrm{~nm}$ (blue), $520 \mathrm{~nm}$ (red), and $750 \mathrm{~nm}$ (black). (a) $T_{\mathrm{TM}}$ vs. $M$ for $270 \leq M \leq 300$ (b) $R_{\mathrm{TM}}$ vs. $M$ for $270 \leq M \leq 300$. (c) $T_{\mathrm{TE}}$ Vs. $M$ for $100 \leq M \leq 300$. (d) $R_{\mathrm{TE}} \mathrm{Vs} . M$ for $100 \leq M \leq 300$.

have fluctuations, as shown in Figs. 4(a) and 4(b). In contrast, the results for the TE cases converge much faster, and their variations in $M>100$ are negligible [Figs. 4(c) and 4(d)]. 


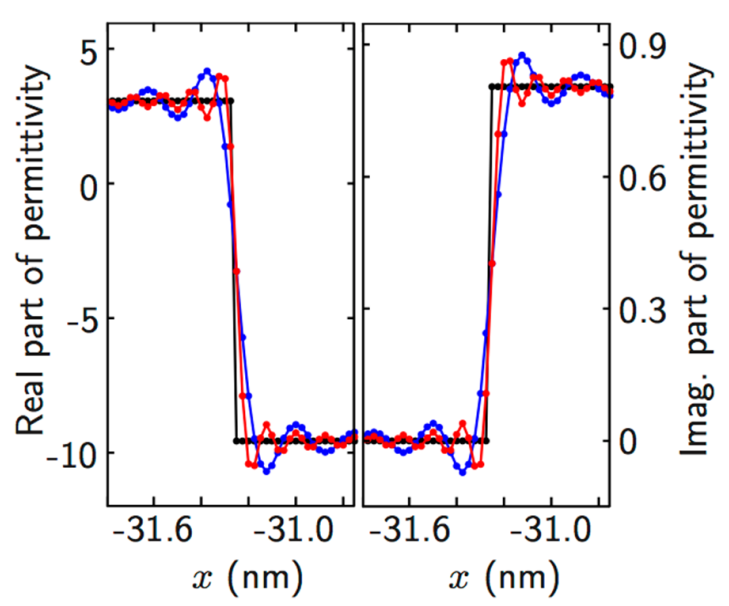

FIG. 5. The exact permittivity profile (black), $\varepsilon(x)$, compared with its Fourier-series approximation, $\hat{\varepsilon}(x)$, with $M=500$ (blue) and 1000 (red). Both real (left) and imaginary (right) parts are shown.

Considering the field profiles shown in Fig. 3, the contrast in convergence characteristics between the TM and TE cases suggests that the fluctuations in $T_{\mathrm{TM}}$ and $R_{\mathrm{TM}}$ are related to the inherent inaccuracies in the Fourier-series approximation of the permittivity profile near the grating boundaries. Figure 5 shows the exact permittivity profile in the grating layer (black), $\varepsilon(x)$, compared with its Fourierseries approximation, $\hat{\varepsilon}(x)$ calculated at $\lambda=520 \mathrm{~nm}$ by Eq. (1) for $M=500$ (blue) and 1000 (red). The $\hat{\varepsilon}(x)$ profiles have oscillations near the grating boundary, and in each (metal or dielectric) region, the oscillation occurring nearest to the grating boundary has the largest magnitude - the characteristic of the Fourier-series representation of a piecewise continuous function with jump discontinuities, known as the Gibbs phenomenon [18]. This observation provides a qualitative and physical explanation that the fluctuations in $T_{\mathrm{TM}}$ and $R_{\mathrm{TM}}$ are, at least in part, ascribed to non-negligible responses of edge-concentrated electromagnetic fields to the fluctuation in $\hat{\varepsilon}(x)$ near discontinuous grating boundaries due to the Gibbs phenomenon. Consequently, $T_{\mathrm{TM}}$ and $R_{\mathrm{TM}}$ show the fluctuations even with $M=270$ and the correct Fourier factorization rules, as shown in Figs. 4(a) and 4(b). Meanwhile, the Gibbs phenomenon does not result in fluctuations in $T_{\mathrm{TE}}$ and $R_{\mathrm{TE}}$, since the extent of regions near the grating boundaries where $\hat{\varepsilon}(x)$ and $\varepsilon(x)$ differ is very small compared with $\Lambda(=250 \mathrm{~nm})$, and the field concentration near the grating boundaries does not occur for TE-polarized illumination [Fig. 3(b)].

\section{ORIGINS OF THE FLUCTUATIONS IN THE CONVERGENCE BEHAVIOR OF THE FMM ANALYSIS}

We examine the numerical framework of the FMM and focus on its internal modal structure to identify the origin of the fluctuations in $T_{\mathrm{TM}}$ and $R_{\mathrm{TM}}$. In the conventional FMM framework, $4(2 M+1)$ Bloch eigenmodes are numerically obtained with $(2 M+1)$ field harmonics, where the coupling between field harmonics is specified by $(4 M+1) \varepsilon_{m}$ 's [1]. Here, it is noted that the number of structure harmonics in Eq. (1) $(=4 M+1)$ and that of field harmonics in Eq. (2) are linked and varied together.

Upon increasing $M$ in the FMM framework, many highly evanescent Bloch eigenmodes are generated, and the degree of evanescence of each mode can be evaluated by the magnitude of the imaginary part of the Bloch eigenvalue $k_{z}^{(g)}$ [1]. By examining the convergence of $k_{z}^{(g)}$ 's with respect to $M$, we find that for given $M$, the calculated $4(2 M+1)$ internal Bloch eigenmodes can be categorized into (i) lower-order non-evanescent or weakly evanescent Bloch modes that are converged, in a sense that their eigenvalues and field profiles are identical to those obtained with smaller $M$ 's, and (ii) a few highly evanescent modes that are not converged yet. The coupling coefficients, in expressing the total field profile as in Eq. (5), of the internal Bloch eigenmodes in categories (i) and (ii) are denoted by $C_{g}^{(i)}$,s, and $C_{g}^{(i)}$ s, respectively.

The convergence of the eigenmodes in category (i) means that $k_{z}^{(g)}$ s and the corresponding field profiles of these eigenmodes are not affected by the oscillating change in $\hat{\varepsilon}(x)$ near the grating edges as $M$ is further increased. Meanwhile, for the eigenmodes in category (ii) to be converged, more higher-order Fourier terms need to be included by increasing $M$. Then, the values of $k_{z}^{(g)}$ for newly generated higher-order terms are different from their converged values; in other words, for any $M$, there are always eigenmodes belonging to category (ii). This finding strongly suggests that the fluctuations in $T_{\mathrm{TM}}$ and $R_{\mathrm{TM}}$ are mainly ascribed to the Bloch eigenmodes in category (ii). More specifically, for TM-polarized illumination, $C_{g}^{(i i)}$, s are not negligible. In viewpoint of the numerical framework, the internal mismatch between the number of field harmonics used $(=2 M+1)$ and that required to accurately represent all $4(2 M+1)$ Bloch eigenmodes causes parameters, such as $T_{\mathrm{TM}}$ and $R_{\mathrm{TM}}$, to exhibit fluctuations. In contrast, $C_{g}^{(i i)}$, $\mathrm{s}$ in the case of TE polarization are negligible, meaning that the unconverged evanescent modes do not contribute to the total field distribution. Consequently, $T_{\mathrm{TE}}$ and $R_{\mathrm{TE}}$ converge without fluctuating features despite the harmonic number mismatch.

To address this issue more clearly, we perform numerical experiments by modifying the conventional FMM framework, where the number of structure harmonics $(=4 M+1)$ representing $\varepsilon(x)$ is directly linked to that of field harmonics (= $2 M+1)$ as seen in Eqs. (1) and (2), to de-couple these two numbers. Specifically, $\varepsilon(x)$ is approximated by

$$
\hat{\varepsilon}_{N}(x)=\sum_{m=-N}^{N} \varepsilon_{m} e^{j m G x}
$$




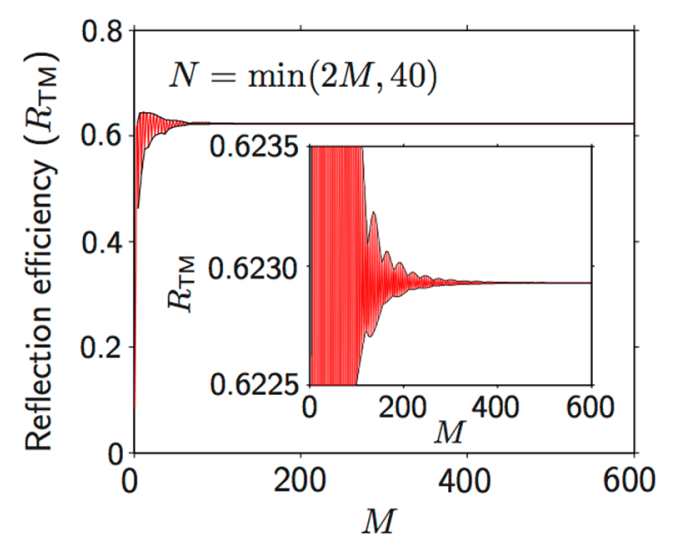

(a)

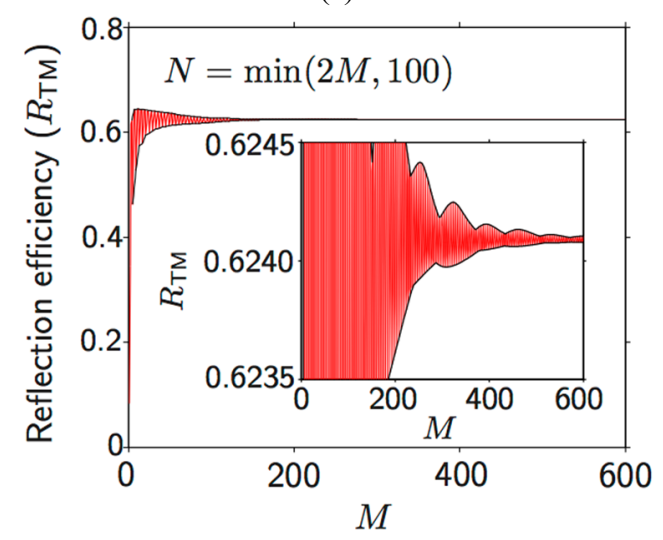

(b)

FIG. 6. Reflection efficiencies (red) versus $M$ when the number of structure harmonics $(2 N+1)$ is limited with (a) $N=$ 40 and (b) $N=100$.

and the electromagnetic fields are given by Eq. (2), where $M$ and $N$ can be independently varied. If $M$ is increased sufficiently beyond the value in the conventional FMM (= $N / 2$ ) for fixed $N$, we expect that the evanescent Bloch eigenmodes with non-negligible coupling coefficients will be converged, and consequently the $T_{\mathrm{TM}^{-}}$and $R_{\mathrm{TM}}-\mathrm{vs} .-M$ plots are expected to converge without fluctuations. This procedure may be considered as band-limiting $\hat{\varepsilon}(x)$ However, we emphasize that it is a means to provide a sufficient number of field harmonics required to accurately represent Bloch eigenmodes involved in diffraction by the approximate grating profile $\hat{\varepsilon}_{N}(x)$.

To show this, we plot $R_{\mathrm{TM}}$ of the one-dimensional grating analyzed in Sec. 3 under $\operatorname{SPR}(\lambda=520 \mathrm{~nm})$ as a function of $M$ with $N$ equal to its value in the convenional FMM $(=2 M)$ only until a certain value (Fig. 6). Figure 6(a) shows the result when the maximum $N\left(\equiv N_{\max }\right)$ is 40 , showing that quick convergence of $R_{\mathrm{TM}}$ is obtained with significantly reduced fluctuations, as evident in the inset. This indicates that if $M>\sim 300, k_{z}^{(g)}$ and the corresponding field profiles of Bloch eigenmodes with non-negligible coupling with the incident wave are completely converged, and this band-limited $\hat{\varepsilon}_{N}(x)$ does not couple the incident

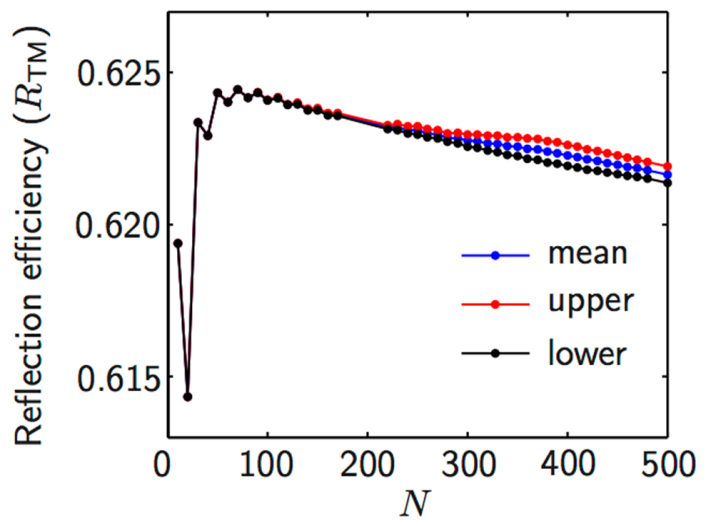

(a)

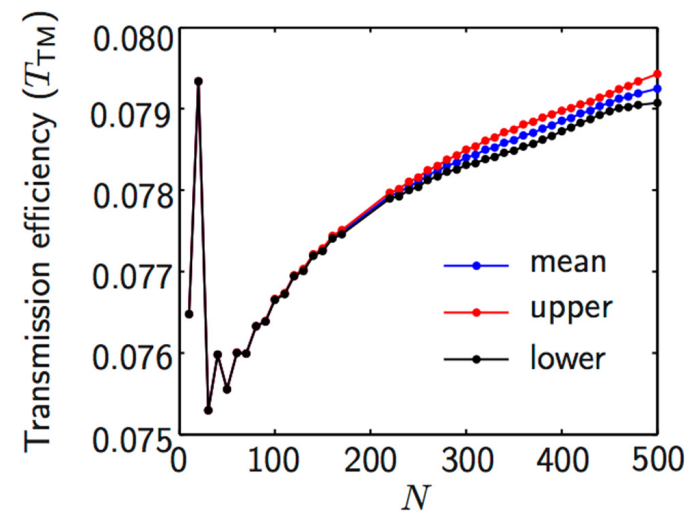

(b)

FIG. 7. (a) Reflection and (b) transmission efficiencies versus $N$, when $M \simeq 600$. For each $N$ envelopes outlining oscillations in $R_{\mathrm{TM}}$ - and $T_{\mathrm{TM}}$-vs. $-M$ plots, such as those shown in Fig. 6, are calculated, and the values of the upper and lower envelopes at $M=600$ are marked as red and black dots. The blue dots represent the mean of the upper and lower envelopes.

wave with the highly evanescent eigenmodes that are not converged yet. Figure 6(b) shows the result for $N_{\max }=100$, where the height of the envelope within which $R_{\mathrm{TM}}$ oscillates becomes larger. We also note that the values of $R_{\mathrm{TM}}$ in Figs. 6(a) and 6(b) converge to different values, since the permittivity profile is slightly different, near the grating boundaries in particular, in each simulation.

These results indicate that calculations of $R_{\mathrm{TM}}$ and $T_{\mathrm{TM}}$ as a function of $N$ for sufficiently large $M$ are free from errors due to unconverged $k_{z}^{(g)}$ of highly evanescent modes, therefore demonstrating fluctuations attributed to the Gibbs phenomenon only. To confirm this, we plot, in Fig. $7, R_{\mathrm{TM}}$ and $T_{\mathrm{TM}}$ of the grating under the same illumination condition as in Fig. 6, as functions of $N$ for $M \simeq 600$ : for each $N$, envelopes outlining oscillations in $R_{\mathrm{TM}^{-}}$and $T_{\mathrm{TM}^{-}} \mathrm{Vs} .-M$ plot, such as those shown in Fig. 6, are calculated, and the values of the upper and lower envelopes at $M=600$ are marked as red and black dots in Fig. 7. Also shown is the mean of the upper and lower envelopes at $M=600$, marked as blue dots. For $N<\sim 200$, the upper and lower 
envelopes of both $R_{\mathrm{TM}}$ and $T_{\mathrm{TM}}$ are indistinguishable, meaning that they are converged with respect to $M$ for each $\hat{\varepsilon}_{N}(x)$. This indicates that $M(=600)$ is sufficiently large to accurately evaluate $k_{z}^{(g)}$ of Bloch eigenmodes whose coupling to the incident plane wave is non-negligible for $\hat{\varepsilon}_{N}(x)$ with $N<\sim 200$, and that the very small fluctuations for $N<\sim 200$ are due to variation in $\hat{\varepsilon}_{N}(x)$. However, as $N$ is increased beyond $\sim 200$, the number of field harmonics $(=2 M+1 \simeq 1200)$ becomes insufficient, resulting in separation between the upper and lower envelopes. This means that both types of errors - one in representing true $\varepsilon(x)$ and the other in evaluating $k_{z}^{(g)}$ of highly evanescent Bloch eigenmodes with non-negligible coupling coefficients contribute to the fluctuations. The magnitude of fluctuations at each $N$ in Fig. 7, i.e., the difference between the upper and the lower envelopes, is much smaller than that of fluctuations around $M=N / 2$ in Fig. 4, which indicates that the errors arising from coupling with unconverged higher order evanescent eigenmodes are reduced in the modified FMM. Although the values of $M$ in our simulations are limited, we infer that if $N$ approaches to infinity while keeping $M \gg N / 2$, the modified FMM will converge with negligible, or much smaller, fluctuations compared to the expected asymptotic behavior of the conventional FMM.

\section{CONCLUSION}

We have unveiled that two types of errors are present in the FMM analysis of a one-dimensional metallic gratings: one in representing true $\varepsilon(x)$ by a Fourier series with a finite number of Fourier terms, which is related to the Gibbs phenomenon, and the other in evaluating field profiles of highly evanescent Bloch eigenmodes. We have found that the number of field harmonics in the FMM framework, $2 M+1$, is always insufficient to accurately represent a few most highly evanescent eigenmodes, and it is an inherent structural limitation of the conventional FMM framework with Li's Fourier factorization rule. We have shown that the fluctuations arising from inaccurate calculations of these eigenmodes can be eliminated in the modified FMM, by increasing $M$ sufficiently beyond the value in the conventional FMM $(=N / 2)$

Due to highly concentrated fields near the grating boundaries, TM-polarized cases are sensitive to this inaccuracy, resulting in fluctuations in $R_{\mathrm{TM}}$ and $T_{\mathrm{TM}}$ even with large $M$. We expect that fluctuations similar to what is observed here to be present in dealing with general metallic structures supporting SPRs, whenever the electric fields are highly concentrated in metal around its discontinuous boundaries. Moreover, in analyzing the electric field enhancement in a deep-subwavelength structure, for example, a metallic slit whose width is significantly smaller than the wavelength of interest [19], the coupling between the excitation field and very-high-order evanescent eigenmodes must be considered.
Since the errors investigated in this paper are the inherent characteristics of the conventional FMM, its application to deep-subwavelength structures can be inaccurate. To obtain accurate results in such cases using the FMM with finite computing resources, the method of representing permittivity profiles may need to be modified. A good strategy may be a form of truncated Fourier series with a specific apodization in the spatial-frequency domain. This topic will be studied based on what is established in this paper.

\section{ACKNOWLEDGMENT}

This work was supported by the Basic Science Research program under Grant Nos. 2011-0026517 and 2010-0022088, and the Engineering Research Center program under Grant No. 2009-0093428, all through the National Research Foundation of Korea, funded by the Ministry of Education, Science and Technology.

\section{REFERENCES}

1. H. Kim, J. Park, and B. Lee, Fourier Modal Method and Its Applications in Computational Nanophotonics (CRC Press, Boca Raton, FL, USA, 2012).

2. P. Lalanne and E. Silberstein, "Fourier-modal methods applied to waveguide computational problems," Opt. Lett. 25, 1092-1094 (2000).

3. Q. Cao, P. Lalanne, and J. Hugonin, "Stable and efficient Bloch-mode computational method for one-dimensional grating waveguides," J. Opt. Soc. Am. A 19, 335-338 (2002).

4. H. Kim, I.-M. Lee, and B. Lee, "Extended scattering-matrix method for efficient full parallel implementation of rigorous coupled-wave analysis," J. Opt. Soc. Am. A 24, 2313-2327 (2007).

5. H. Kim and B. Lee, "Mathematical modeling of crossed nanophotonic structures with generalized scattering- matrix method and local Fourier modal analysis," J. Opt. Soc. Am. B 25, 518-544 (2008).

6. C.-H. Sun, P. Jiang, and B. Jiang, "Broadband moth-eye antireflection coatings on silicon," Appl. Phys. Lett. 92, 061112 (2008).

7. C. Mateus, M. Huang, Y. Deng, A. Neureuther, and C. ChangHasnain, "Ultrabroadband mirror using low-index cladded subwavelength grating," IEEE Photon. Technol. Lett. 16, 518-520 (2004).

8. Y. Ding and R. Magnusson, "Resonant leaky-mode spectralband engineering and device applications," Opt. Express 12, 5661-5674 (2004).

9. S. B. Mallick, N. P. Sergeant, M. Agrawal, J.-Y. Lee, and P. Peumans, "Coherent light trapping in thin-film photovoltaics," MRS Bull. 36, 453-460 (2011).

10. E. Silberstein, P. Lalanne, J. Hugonin, and Q. Cao, "Use of grating theories in integrated optics," J. Opt. Soc. Am. A 18, 2865-2875 (2001).

11. D. Carr, J. Sullivan, and T. Friedmann, "Laterally deformable nanomechanical zeroth-order gratings: anomalous diffraction 
studied by rigorous coupled-wave analysis," Opt. Lett. 28, 1636-1638 (2003).

12. L. Li, "Use of Fourier series in the analysis of discontinuous periodic structures," J. Opt. Soc. Am. A 13, 1870-1876 (1996).

13. P. Lalanne and G. Morris, "Highly improved convergence of the coupled-wave method for TM polarization," J. Opt. Soc. Am. A 13, 779-784 (1996).

14. G. Granet and B. Guizal, "Efficient implementation of the coupled-wave method for metallic lamellar gratings in TM polarization," J. Opt. Soc. Am. A 13, 1019-1023 (1996).

15. H. Liu and P. Lalanne, "Microscopic theory of the extraordinary optical transmission," Nature 452, 728-731 (2008).
16. K. Koerkamp, S. Enoch, F. Segerink, N. van Hulst, and L. Kuipers, "Strong influence of hole shape on extraordinary transmission through periodic arrays of subwavelength holes," Phys. Rev. Lett. 92, 183901 (2004).

17. E. D. Palik, Handbook of Optical Constants of Solids (Academic Press, NY, USA, 1997).

18. G. P. Tolstov, Fourier Series (Dover Publications, Mineola, NY, USA, 1976)

19. M. A. Seo, H. R. Park, S. M. Koo, D. J. Park, J. H. Kang, O. K. Suwal, S. S. Choi, P. C. M. Planken, G. S. Park, N. K. Park, Q. H. Park, and D. S. Kim, "Terahertz field enhancement by a metallic nano slit operating beyond the skin-depth limit," Nat. Photonics 3, 152-156 (2009). 